\title{
Shp2 regulates chlorogenic acid-induced proliferation and adipogenic differentiation of bone marrow-derived mesenchymal stem cells in adipogenesis
}

\author{
RONG-PING ZHOU ${ }^{1 *}$, MING-TAO DENG ${ }^{1 *}$, LAN-YING CHEN $^{2}$, NING FANG $^{1}$, CHUAN DU $^{1}$, \\ LIN-PAN CHEN $^{1}$, YE-QING ZOU ${ }^{1}$, JIANG-HUA DAI ${ }^{1}$, MEI-LAN ZHU ${ }^{1}$, WEI WANG ${ }^{1}$, \\ SI-JIAN LIN ${ }^{1}$, RONG-HUA LIU ${ }^{2}$ and JUN LUO ${ }^{1}$ \\ ${ }^{1}$ Orthopaedic Department, The Second Affiliated Hospital of Nanchang University, Nanchang, Jiangxi 330006; \\ ${ }^{2}$ Pharmacy College, Jiangxi University of Traditional Chinese Medicine, Nanchang, \\ Jiangxi 330004, P.R. China
}

Received March 12,2014; Accepted December 17, 2014

DOI: $10.3892 / \mathrm{mmr} .2015 .3285$

\begin{abstract}
Chlorogenic acid (CGA) exhibits various biological properties, including the inhibition of oxidation, obesity, apoptosis and tumorigenesis. CGA is also able to promote cell survival and proliferation. The aim of the present study was to determine the effects and underlying molecular mechanisms of CGA on the adipogenesis of bone marrow-derived mesenchymal stem cells (BMSCs). Treatment with CGA had a marginal effect on cell proliferation, by promoting the expression levels of phosphorylated Akt and cyclin D1. Furthermore, treatment with CGA also upregulated the phosphorylation of extracellular signal-regulated kinase (Erk) and inhibited the adipocyte differentiation of BMSCs by inhibiting the expression of peroxisome proliferator-activated receptor $(\mathrm{PPAR}) \gamma$ and CCAAT/enhancer binding protein $(\mathrm{C} / \mathrm{EBP}) \alpha$. However, knockdown of the expression of Shp2 attenuated CGA-induced proliferation and inhibited the phosphorylation of Akt and expression of cyclin D1. Furthermore, CGA treatment upregulated Erk phosphorylation and decreased the expression levels of PPAR $\gamma$ and CEBP $\alpha$, which was inhibited by treatment with the Shp2 PTPase activity inhibitor, NSC-87877. The results of the present study suggested that
\end{abstract}

Correspondence to: Professor Jun Luo, Orthopaedic Department, The Second Affiliated Hospital of Nanchang University, 1 Minde Road, Nanchang, Jiangxi 330006, P.R. China

E-mail: luojun1786@163.com

Professor Rong-Hua Liu, Pharmacy College, Jiangxi University of Traditional Chinese Medicine, 818 Xingwan Road, Nanchang, Jiangxi 330004, P.R. China

E-mail:rhliu@163.com

*Contributed equally

Key words: chlorogenic acid, bone mesenchymal stem cell, Shp2, proliferation, differentiation
CGA-induced Akt and Erk pathways regulate proliferation and differentiation and that Shp2 is important in the proliferation and differentiation of BMSCs.

\section{Introduction}

The multipotent nature of bone marrow-derived mesenchymal stem cells (BMSCs) suggests that they may be potentially applicable to clinical cell therapy. However, the limited number of BMSCs, which constitute $\sim 0.001 \%$ of the total bone marrow cells, presents a challenge for future stem cell therapy using these cells $(1,2)$.

Chlorogenic acid (CGA) is a chemical monomer with antitumor effects, which is present in various types of plants, including Eucommia (3). CGA also prevents obesity by inhibiting the release of glucose into the blood (4). A previous study demonstrated that CGA increases the phosphorylation of Akt in response to apoptotic stimuli (5). Shp2 is a cytoplasmic tyrosine phosphatase and is a critical controlling factor in the interaction of the phospohinositide 3-kinase (PI3K) and mitogen-activated protein kinase (MAPK) signaling pathways $(6,7)$. Shp2-mediated molecular signaling pathways, including extracellular signal-regulated kinases (Erk) $1 / 2$ and PI3K/Akt, are associated with the regulation of stem cell differentiation and proliferation $(8,9)$.

The present study investigated the mechanisms underlying CGA-induced BMSC proliferation and inhibition of adipocyte differentiation. To the best of our knowledge, the mechanisms underlying CGA-induced BMSC proliferation and inhibition of adipocyte differentiation have not previously been reported. The preent study aimed to elucidate whether, in response to treatment with CGA, the Shp2, PI3K/Akt and cyclin D1 signaling pathways were involved in BMSC proliferation, and the Shp2, MAPK/Erk, peroxisome proliferator-activated receptor (PPAR) $\gamma$ and CCAAT/ enhancer binding protein (C/EBP) $\alpha$ pathways were involved in the inhibition of adipocyte differentiation. The results may aid the determination of whether CGA may offer potential benefits in tissue engineering and provide a novel theoretical 
basis for innovative strategies in future clinical applications; and furthermore, may clarify the mechanisms underyling the effects of CGA in the prevention and treatment of obesity.

\section{Materials and methods}

Cell culture. Bone marrow aspirates were obtained from iliac crest biopsies of two healthy donors (male, 26 years old, left calcaneus fracture and female, 32 years old, right plateau fracture, required open reduction and internal fixation, bone graft) during routine surgical procedures. The bone marrow was obtained during the bone graft procedures. The present study was approved by the Ethical Committee of the Second Affiliated Hospital of Nanchang University (Nanchang, China) and all the patients provided informed consent. Mononucleated cells were isolated from the bone marrow by density gradient centrifugation at $1,000 \mathrm{x} \mathrm{g}$ for 15 min using human lymphocyte separation medium (density $1.077 \pm 0.0001 \mathrm{~g} / \mathrm{ml}$; Beijing Solarbio Science \& Technology, Co., Ltd., Beijing, China). The cells were subsequently seeded and grown in Low Glucose Dulbecco's modified Eagle's medium (DMEM; Hyclone Laboratories, Inc., Logan, UT, USA), supplemented with $15 \%$ fetal bovine serum (FBS; Hyclone Laboratories, Inc.), at $37^{\circ} \mathrm{C}$ in a humidified atmosphere containing $5 \% \mathrm{CO}_{2} / 95 \%$ air. BMSCs were observed under a phase contrast microscope (IX71; Olympus Corp., Guangzhou, China).

To induce adipogenesis, the BMSCs were seeded at a density of $1 \times 10^{4} \mathrm{cell} / \mathrm{cm}^{2}$ in 24 -well plates (Corning Incorporated, Corning, NY, USA). Following a $24 \mathrm{~h}$ culture, the cells were treated with adipogenic medium, consisting of growth medium supplemented with StemPro ${ }^{\circledR}$ Adipogenesis Differentiation kit (Gibco Life Technologies, Carlsbad, CA, USA). After 14 days, the adipogenesis was visualized by light microscopy (IX71; Olympus Corp.), with or without staining by Oil Red O (Bogu Biotechnology Co., Ltd, Shanghai, China), as described previously $(10,11)$. Subsequently, the BMSCs were treated with $0,0.1,1,10$ or $100 \mu \mathrm{M}$ CGA (\#C3878; Sigma-Aldrich, St. Louis, MO, USA) for $15 \mathrm{~min}$, and the expression levels of markers, including Erk1/2, PPAR $\gamma$ and CEBP $\alpha$, were assessed by reverse transcription quantitative polymerase chain reaction (RT-qPCR) and western blotting. Furthermore, the expression levels of the signaling pathways, which regulate cell proliferation, including PI3K/Akt and cyclin D1, were determined by RT-qPCR and western blotting in the BMSCs treated with $0.1,1,10$ or $100 \mu \mathrm{M}$ CGA (treated group) or $0 \mu \mathrm{M}$ CGA (control group) for $48 \mathrm{~h}$.

Western blot analysis. The cells were washed twice with phosphate-buffered saline (PBS; Hyclone; Thermo Fisher Scientific, Rockford, IL, USA) and then lysed on ice with radioimmunoprecipitation buffer $(50 \mathrm{mM}$ Tris, $150 \mathrm{mM} \mathrm{NaCl}$, $1.0 \%$ NP-40, $0.5 \%$ sodium deoxycholate and $0.1 \%$ SDS; $\mathrm{pH}$ 7.4) supplemented with $10 \mu \mathrm{l} / \mathrm{ml}$ protease inhibitor cocktail (cat. no. 11836170001) and PhosStop (one tablet per $10 \mathrm{ml}$; cat no. \#4906845001; Roche, Nutley, NJ, USA). The protein content was quantified using a Bicinchoninic Acid Protein Assay kit (Pierce Biotechnology, Inc., Rockford, IL, USA), according to the manufacturer's instructions. For western blotting, $30-50 \mu \mathrm{g}$ of the protein samples were separated by $10 \%$ SDS-PAGE $\left[\mathrm{H}_{2} \mathrm{O}, 30 \%\right.$ acrylamide, $1.5 \mathrm{MTris}-\mathrm{HCl}$ $(\mathrm{pH} 8.8), 10 \%$ SDS, $10 \%$ ammonium persulfate and tetramethylethylenediamine] and transferred onto polyvinylidene difluoride membranes (Ruiqi Biotechnology Co., Ltd, Shanghai, China). The membranes were then incubated for $8-12 \mathrm{~h}$ at $4-8^{\circ} \mathrm{C}$ with the following primary antibodies, purchased from Cell Signaling Technology, Inc. (Danvers, MA, USA): Rabbit polyclonal phosphorylated (p)-SHP-2 (Tyr580; cat. no. \#5431), rabbit monoclonal p-p44/42 MAPK (T202/Y204; D13.14.4E; cat. no. 4370S), rabbit monoclonal p-Akt (ser473; cat. no. 4060S), mouse monoclonal cyclin D1 (cat. no. 2926), and Santa Cruz Biotechnology, Inc.: Rabbit polyclonal SH-PTP2 (cat. no. sc-280), rabbit polyclonal Akt1/2/3 (H-136; cat. no. sc-8312) and mouse monoclonal $\beta$-Actin (C4; cat. no. sc-47778). Primary antibodies $(1: 1,000)$ and horseradish-peroxidase-conjugated secondary antibodies (1:1,000; HRP-labeled Goat Anti-Rabbit IgG(H+L), A0208 and HRP-labeled Goat Anti-Mouse IgG(H+L), A0216, Beyotime, Nanjing, China) were diluted with Tris-buffered saline-0.1\% Tween ${ }^{\circledR} 20(100 \mathrm{mmol} / 1$ Tris $\mathrm{HCl}, \mathrm{pH} 7.5$; $150 \mathrm{mmol} / \mathrm{l} \mathrm{NaCl}$ ), containing $5 \%$ bovine serum albumin (\#A7906; Sigma-Aldrich). Membranes were incubated with secondary antibodies for 1-2 $\mathrm{h}$ at room temperature (12). The immunoreactive signals were then visualized using the Enhanced Chemiluminescence Plus system (Zhongshan Biotechnology Co., Ltd, Beijing, China).

Inhibitor studies and small interfering (si)RNA-mediated suppression of Shp2. An ERK inhibitor (10 $\mu \mathrm{M}$ PD98059; \#9900; Cell Signaling Technology, Inc.) and PI3K inhibitor (0.1 $\mu \mathrm{M}$ Ly294002; \#S1105; Selleck, Shanghai, China) were added to the cells $\left(1 \times 10^{5}\right.$ cells $\left./ \mathrm{ml}\right)$ at $37^{\circ} \mathrm{C}, 2 \mathrm{~h}$ prior to the addition of CGA. siRNA specific to Shp2, forward 5'-GAAUAUGGCGUCAUGCGUGTT-3' and reverse 5'-C ACGCAUGACGCCAUAUUCTT-3' (13) and scrambled siRNA, forward 5'-AGUUAUAAGGCGGUCGUGCTT-3' and reverse 5'-GCACGACCGCCUUAUAACUTT-3' were synthesized by Invitrogen Life Technologies (Carlsbad, CA, USA). The siRNAs were transfected into the BMSCs using Lipofectamine ${ }^{\circledR} 2000$ reagent (Invitrogen Life Technologies) (14) for $6 \mathrm{~h}$, and the cells were recovered in DMEM supplemented with $10 \%$ FBS. The cells were then harvested and reseeded at $1 \times 10^{5}$ cells $/ \mathrm{ml}$ in 96 -well plates for subsequent bromodeoxyuridine (BrdU) and RT-qPCR assays.

BrdU assay. The BrdU assay was performed using a Cell Proliferation kit (cat. no. \#2750; Merck Millipore, Darmstadt, Germany), according to the manufacturer's instructions. The incorporation of BrdU was detected by horseradish-peroxidase substrate (\#S131125; Tiandz, Inc., Beijing, China) and the color intensity was measured at $450 \mathrm{~nm}$ (ELx800; Bio-Tek Instruments, Inc., Winooski, VT, USA).

$R T-q P C R$. RT-qPCR was performed, as described in our previous study (5). The PCR conditions used were as follows: $95^{\circ} \mathrm{C}$ for $3 \mathrm{~min}$, followed by 40 cycles of $95^{\circ} \mathrm{C}$ for $20 \mathrm{sec}, 52^{\circ} \mathrm{C}$ for $30 \mathrm{sec}$ and $60^{\circ} \mathrm{C}$ for $30 \mathrm{sec}$. The results were analyzed using the cycle threshold $(\mathrm{Ct})$ relative quantification method $\left({ }^{\Delta \Delta} \mathrm{Ct}\right)$, using the iQ5 Optical System Software version 2.0 

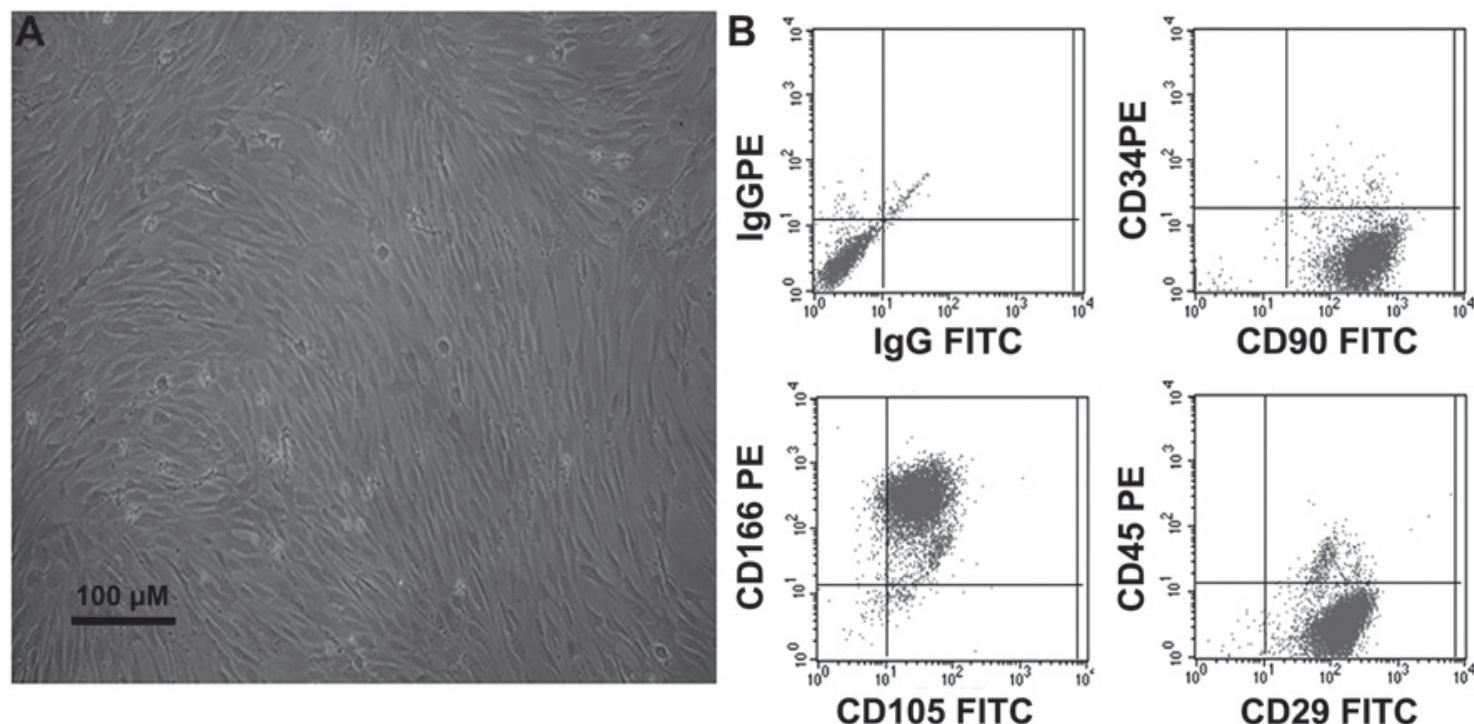

Figure 1. (A) BMSCs were observed under phase-contrast microscopy. (B) BMSC phenotype was determined by flow cytometric analysis. The BMSCs expressed CD29, CD90, CD105 and CD166 markers, but were negative for CD34 and CD45. BMSC, bone marrow-derived stem cell; CD, cluster of differentiation; FITC, fluorescein isothiocyanate; PE, phycoerythrin; IgG, immunoglobulin G.

(Bio-Rad Laborotories, Inc., Hercules, CA, USA). The Ct values were obtained using ABI 7300 software (Applied Biosysystems Life Technologies, Foster City, CA, USA). The fold change of the relative expression of mRNA was determined using the $2^{-\Delta \Delta \mathrm{Ct}}$ method (15).

Flow cytometry. The BMSC suspensions were washed twice with PBS. For direct assays, $5 \times 10^{4}$ cells were incubated with $1 \mathrm{mg} / \mathrm{ml}$ (1/1,000 dilution) fluorescein isothiocyanate-conjugated CD29, CD90 and CD105, or phycoerythrin-conjugated CD34, CD45 and CD166 (\#ab81289, \#ab10925 and \#ab93758; Abcam, Shanghai, China) in the dark for $15 \mathrm{~min}$, and were analyzed by flow cytometric analysis using a fluorescence-activated cell sorting (FACS)Calibur flow cytometer (BD Biosciences, San Jose, CA, USA) with the use of CellQuest software (TreeStar.FlowJo.v5.7; BD Biosciences, San Jose, CA, USA).

Statistical analysis. All the data are expressed as the mean \pm standard deviation. One-way analysis of variance, followed by Student's t-test were used to determine statistically significant differences between the experimental and control groups. Statistical analyses were conducted using SPSS 13.0 (SPSS Inc., Chicago, IL, USA). P $<0.05$ was considered to indicate a statistically significant difference (5).

\section{Results}

Characterization of undifferentiated BMSCs. Following 2-3 days of culture, the non-adherent cells were removed by replacing the low glucose DMEM, and the attached nucleated cells were visualized. These cells displayed an elongated fibroblast-like morphology under phase-contrast microscopy (Fig. 1A). Furthermore, the immunophenotypes of the expanded clonal BMSCs were analyzed by FACS. The BMSCs expressed the CD29, CD90, CD105 and CD166 markers, but not the CD34 or CD45 markers (Fig. 1B). This phenotype remained unchanged for $>20$ passages, consistent with the results observed in a previous study by our group (5).

Treatment with CGA induces the proliferation of BMSCs via PI3K/Akt/cyclin D1 signaling. The effects of CGA on the proliferation of BMSCs was investigated using a BrdU assay. Cell proliferation was significantly enhanced following treatment with CGA at doses between 0.1 and $10 \mu \mathrm{M}$, compared with the control group and the group treated with $100 \mu \mathrm{M} \mathrm{CGA}$ (Fig. 2A). Cell death was observed following treated of the cells with $100 \mu \mathrm{M}$ CGA. To determine the mechanism involved in the proliferation of BMSCs, the effects of CGA on the growth of BMSCs, cultured in phenol red-free medium containing $1 \%$ FBS, were investigated, at $48 \mathrm{~h}$. Alterations in the phosphorylation of Akt and the expression of cyclin D1 in response to stimulation with CGA, were determined. The phosphorylation of Akt and the protein expression levels of cyclin D1 were increased in response to treatment with CGA at concentrations of $0.1,1$ and $10 \mu \mathrm{M}$ (Fig. 2B); however, the phosphorylation of Erk was not enhanced by treatment with CGA at these concentrations (data not shown). In addition, the inhibition of PI3K/Akt, by the specific inhibitor, LY294002, reduced the proliferation of cells and deceased the expression levels of cyclin D1 (Fig. 2C and D).

Shp2 is a regulator of CGA/PI3K/Akt and induces BMSC proliferation. Shp2 is a major signal cytoplasmic tyrosine phosphatase and contains two $\mathrm{SH} 2$ domains upstream of PI3K/Akt/cyclin D1, which have been implicated in mitogenic responses (16). Therefore, the present study investigated whether Shp2 was also a regulator of CGA-induced BMSC proliferation. The expression levels of Shp2 were suppressed in the BMSCs, which were transfected with siRNA. The proliferation of BMSCs was inhibited and activation of the $\mathrm{PI} 3 \mathrm{~K} / \mathrm{Akt} /$ cyclin D1 pathways was induced by the combination of siRNA and Shp2 (10 nM), compared with the mock-transfected cells (Fig. 3). 


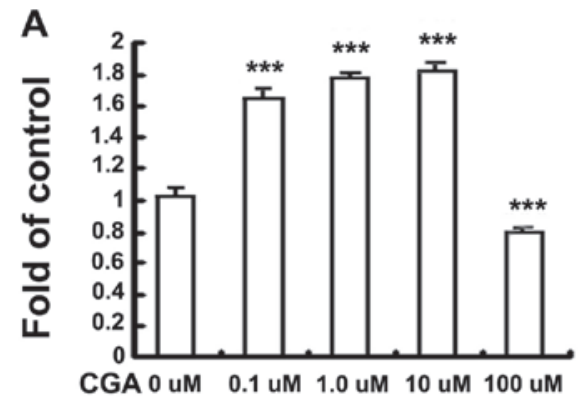

B

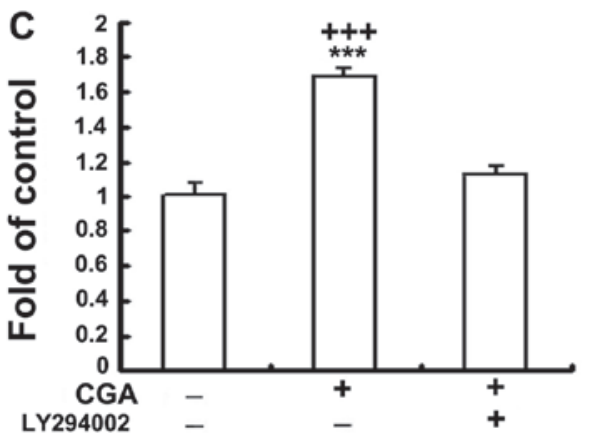

D

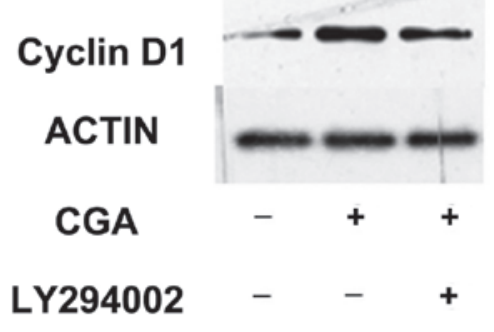

Figure 2. (A) Effects of CGA $(0.1-100 \mu \mathrm{M})$ on the proliferation of BMSCs was investigated using a BrdU assay. BMSCs were seeded with serum-free medium, without phenol red, and starved for $6 \mathrm{~h}$, followed by treatment with $0,0.1,1,10$ or $100 \mu \mathrm{M}$ CGA for $48 \mathrm{~h}$. The proliferation rate was determined by measuring fluorescence intensity at $450 \mathrm{~nm}$. BrdU results (means \pm standard error; $\mathrm{n}=3$ ) indicate cellular BrdU incorporation into DNA in arbitrary units. (B) BMSCs were seeded with serum-free medium (1\%) without phenol red, and starved for $6 \mathrm{~h}$, followed by treatment with $0,0.1,1.0$ or $10 \mu \mathrm{M} \mathrm{CGA}$ for $48 \mathrm{~h}$. The protein expression levels of phosphorylated Akt and cyclin D1 were detected by western blotting. (C) BMSCs were serum deprived (1\%) without phenol red, for $6 \mathrm{~h}$ and subsequently cultured in $1 \mu \mathrm{M} \mathrm{CGA}$ in the presence or absence of LY294002 $(20 \mu \mathrm{M})$ for $48 \mathrm{~h}, 2 \mathrm{~h}$ prior to stimulation with CGA. Proliferation was determined by measuring fluorescence intensity at $450 \mathrm{~nm}$. (D) BMSCs were serum deprived (1\%) without phenol red, for $6 \mathrm{~h}$ and subsequently cultured in $1 \mu \mathrm{M} \mathrm{CGA}$ in the presence or absence of LY294002 $(20 \mu \mathrm{M})$ treatment for $48 \mathrm{~h}, 2 \mathrm{~h}$ prior to stimulation with CGA. The protein expression levels of p-Akt and cyclin D1 were detected by western blotting. BMSCs, bone marrow-derived stem cells; CGA, chlorogenic acid; BrdU, bromdeoxyuridine; p-, phosphorylated.
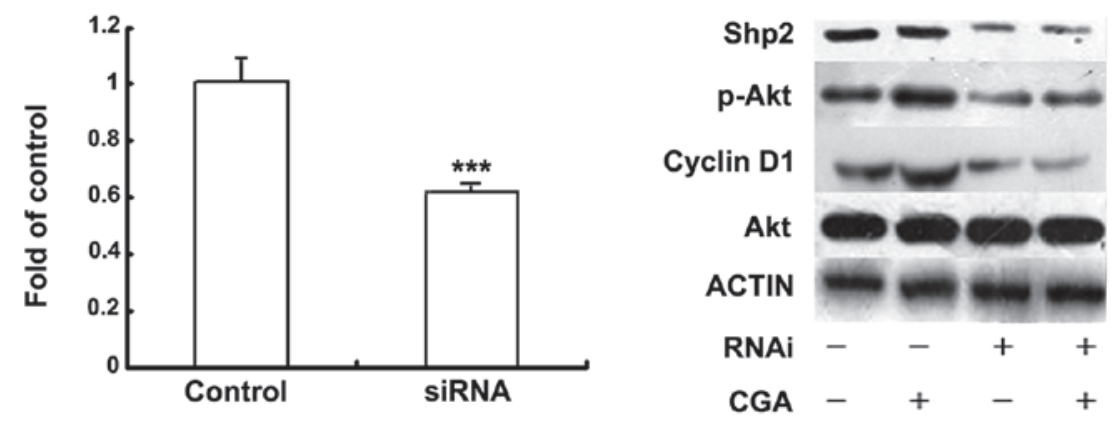

Figure 3. Bone marrow-derived stem cells were grown to 70-80\% confluence and were then transfected with Shp2 or control (scrambled) siRNA for $6 \mathrm{~h}$ using Lipofectamine ${ }^{\circledR} 2000$. The cells were then recovered in Dulbecco's modified Eagle's medium supplemented with $10 \%$ fetal bovine serum. A BrdU assay and western blotting were performed following culture of the cells with or without $1 \mu \mathrm{M}$ CGA for $48 \mathrm{~h}$. BrdU results (means \pm standard error, $\mathrm{n}=3$ ) indicate the cellular BrdU incorporation into DNA, in arbitrary units. BrdU, bromodeoxyuridine; CGA, chlorogenic acid; siRNA, small interfering RNA; RNAi, RNA interference.
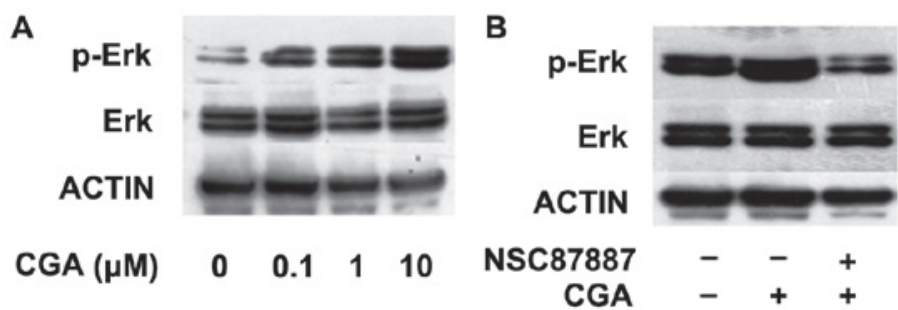

Figure 4. (A) BMSCs were grown in serum-free medium (1\%) without phenol red and starved for $6 \mathrm{~h}$, followed by treatment with $0,0.1,1$ or $10 \mu \mathrm{M}$ CGA for $15 \mathrm{~min}$. The protein expression levels of phosphorylated Erk were detected by western blotting. (B) BMSCs were serum deprived (1\%) without phenol red for $6 \mathrm{~h}$ and subsequently cultured in $1 \mu \mathrm{M} \mathrm{CGA}$, in the presence or absence of NSC87887 $(10 \mu \mathrm{M})$ for $15 \mathrm{~min}, 2 \mathrm{~h}$ prior to stimulation with CGA. The protein expression levels of phosphorylation of Erk were detected by western blotting. BMSC, bone marrow-derived stem cells; CGA, chlorogenic acid; Erk, extracellular signal-regulated kinase; $p$, phosphorylated. 

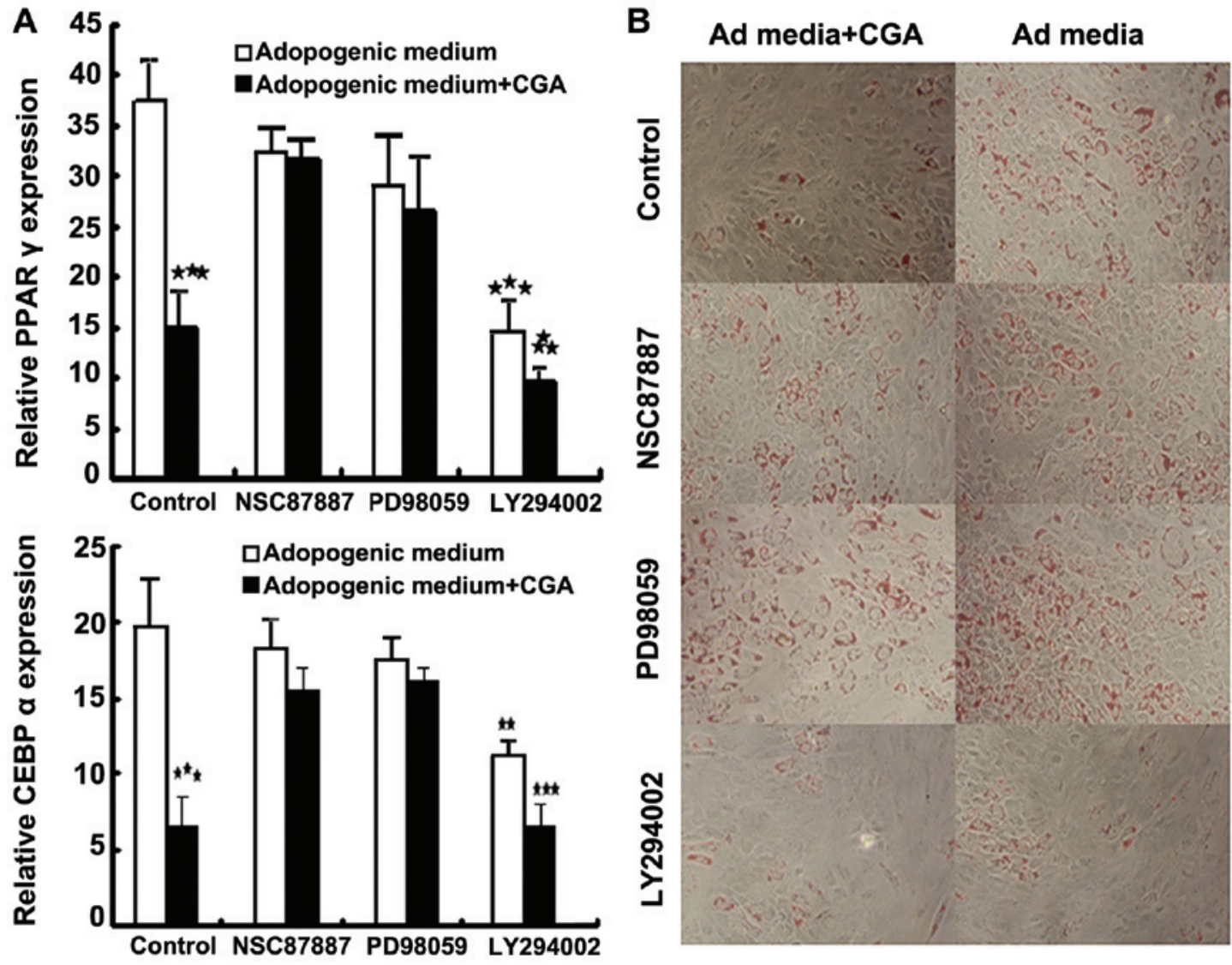

Figure 5. Bone marrow-derived stem cells were incubated with Ad medium, with or without LY294002 (20 $\mu \mathrm{M})$, PD98059 (20 $\mu \mathrm{M})$ or NSC87887 (10 $\mu \mathrm{M})$, in the presence or absence of $1 \mu \mathrm{M}$ CGA for 7 days. (A) mRNA expression levels of the adipogenic markers, PPAR $\gamma$ and CEBP $\alpha$, were analyzed by reverse transcription quantitative polymerase chain reaction (mean \pm standard error of the mean of three experiments of duplicates). ${ }^{* *} \mathrm{P}<0.01$ and ${ }^{* * * *} \mathrm{P}<0.001$, compared with the cells cultured in differentiation medium alone. (B) In order to assess phenotypic changes, the cells were differentiated for a further 7 days. Lipid accumulation in the treated and untreated populations was visualized by Oil Red O staining (magnification, x100). CGA, chlorogenic acid; CEBP $\alpha$, CCAAT/enhancer binding protein- $\alpha$; PPAR $\gamma$; peroxisome proliferator-activated receptor- $\gamma$; Ad, adipogenic.

CGA-induced regulation of BMSC differentiation occurs via Shp2/Erk signaling. The activation or inhibition of the PI3K/Akt and Erk signaling pathways has previously been observed to regulate the adipogenic differentiation of pre-adipocyte cell lines $(17,18)$. However, the role of CGA in the adipogenesis of BMSCs and the underlying mechanisms remain to be elucidated. The present study assessed the effect of Shp2 inhibition on the Erk and Akt signaling pathways on BMSC adipogenic differentiation, in the absence or presence of CGA. The phosphorylation of Erk was significantly enhanced following treatment with CGA, at a dose between 0.1 and $10 \mu \mathrm{M}$, compared with the control group at 15 min (Fig. 4A); however, this effect was inhibited by treatment with the NSC-87877 Shp2 PTPase activity inhibitor (Fig. 4B).

Notably, treatment with NSC87887 and PD98059 had little effect on the adipogenesis of BMSCs in differentiation medium, however, increases in the expression levels of PPAR $\gamma$ and CEBP $\alpha$ were detected (Fig. 5A). The addition of LY294002 to the differentiation medium decreased the number of lipid-containing adipocytes, and significantly inhibited the expression levels of PPAR $\gamma$ and CEBP $\alpha$ (Fig. 5A). The activation of CGA and inhibition of Akt resulted in reduced adipogenic differentiation of the BMSCs (Fig. 5B). These results suggested that other downstream pathways, rather than Akt, were involved. As shown in Fig. 5, NSC87887 and PD98059 inhibited the inhibition of adipogenic differentiation of BMSCs in the presence of CGA. Therefore, treatment with CGA inhibited the adipogenic differentiation of BMSCs and the expression of PPAR $\gamma$ and CEBP $\alpha$ by enhancing the phosphorylation of Erk, but not Akt.

\section{Discussion}

MSCs have received significant attention due to their various applications in regenerative medicine. Understanding the pathways controlling the fate of MSCs is likely to benefit in vivo and in vitro tissue engineering approaches. MSCs isolated from aged animals and humans exhibit reduced proliferative and differentiation capacities (19). In addition, the rate of malignancy is higher in aged stem cells and the molecular pathways underlying tumorigenesis and proliferation of stem cells are the same (20-22). CGA, an antioxidant and metal chelator, has been reported to have antimicrobial (23), anti-inflammatory (24) and glycemic $(25,26)$ effects. Furthermore, the preventive role of CGA in human colon cancer (27), oral tumor cell lines (28), and murine preadipocytes (29) has been demonstrated.

The present study revealed that treatment with CGA promoted the proliferation of BMSCs and inhibited the 
adipocyte differentiation of BMSCs, of which Shp2 was identified as a key regulator. Major signaling pathways, including PI3K/Akt and cyclin D1, but not MEK/Erk, were partly involved in the growth of BMSCs, as only inhibition of the PI3K/Akt pathway was able to reverse the stimulatory effects of CGA on MSC proliferation. Notably, only the activity of Akt was sustained throughout the investigation, whereas the phosphorylation of Erk was rapid and transient. It is well known that the duration of these pathways determines the subsequent physiological response of the cells. Sustained Erk activation by fibroblast growth factor-2 has been observed to induce the proliferation of MSCs and dermal fibroblasts $(30,31)$, whilst the transient activation of Erk has been associated with cell differentiation (32).

The mechanisms by which the activation of Akt or Erk leads to cell proliferation or differentiation differ depending on the type of cell $(33,34)$. The present study demonstrated that CGA had a proliferative effect on BMSCs by promoting cyclin D1. In correlation with the cell proliferation data, the stimulation of regulation by CGA was inhibited by Akt and Shp2 inhibitors. Although the PI3K/Akt pathway was the principal mediator of CGA-induced MSC proliferation, it was not involved in the regulation of CGA-induced MSC differentiation. This was supported by the observation that LY294002 markedly inhibited the adipogenesis of MSCs, which was induced by differentiation medium in the absence or presence of CGA. Furthermore, the addition of CGA to normal medium induced the expression of the adipogenic 'master switch'-PPAR $\gamma$ (35). These results suggested a role for CGA-induced Akt in the proliferation of MSCs, but not in cell differentiation. Notably, although Erk was activated by CGA signaling in the MSCs, it had a negligible effect on proliferation and was found to be responsible for the action of adipogenic medium on adipogenic differentiation. The ability of CGA to inhibit adipogenesis through Erk appeared only when the cells were induced to differentiate. In the undifferentiated cells, CGA either upregulated or had no effect on the expression of the genes involved in adipogenesis. Based on previous data, Akt is required and sufficient for adipogenesis, suggesting that the upregulation of Akt may lead to differentiation and proliferation $(36,37)$. However, the simultaneous activation of Erk inhibits adipogenesis and maintains cells in an undifferentiated state. Erk signaling has previously been implicated in adipogenesis and to have a negative effect on adipocytes; however, stimulation was reported to be dependent on the upstream signal $(11,17,38)$. These findings indicate that self-renewal of MSCs requires a complex interaction between various signaling pathways. In the present study, the Akt pathway promoted MSC proliferation, and Erk signaling was required for maintaining cells in an undifferentiated state, countering possible adipogenic differentiation through activation of Akt. This may explain why Akt induces adipogenic differentiation and causes spontaneous differentiation in preadipocyte 3T3L1 cells when activated either by overexpression of Akt or by factors, including insulin (17). However, this was not observed for CGA with MSCs.

In conclusion, the results of the present study demonstrated that CGA maintained the self-renewal of BMSCs by inducing proliferation and preventing cell differentiation. Treatment with CGA simultaneously activated two major transduction pathways, with dual actions on proliferation and differentiation. When the BMSCs were treated with CGA, the Shp2, PI3K/Akt and cyclin D1 signaling pathways were found to be involved in BMSC proliferation, whilst the Shp2, MAPK/Erk, peroxisome proliferator-activated receptor (PPAR) $\gamma$ and CCAAT/enhancer binding protein $(\mathrm{C} / \mathrm{EBP}) \alpha$ pathways were involved in the inhibition of adipocyte differentiation. Therefore, CGA may activate PI3K/Akt, as the mediator of cell proliferation, and Erk, as the inhibitor of differentiation, in MSCs. It is clear that the effects of CGA avoid unwanted differentiation whilst MSCs undergo extensive proliferation. These findings may contribute to the development of novel strategies for the regulation of stem cell proliferation and differentiation, which may be used in future clinical applications.

\section{Acknowledgements}

The present study was supported by the National Natural Science Foundation of China (nos. 81160508, 81260401 and 81160226), and the Natural Science Foundation of Jiangxi Province (no. GZY0170).

\section{References}

1. Wong KL, Lee KB, Tai BC, Law P, Lee EH and Hui JH: Injectable cultured bone marrow-derived mesenchymal stem cells in varus knees with cartilage defects undergoing high tibial osteotomy: a prospective, randomized controlled clinical trial with 2 years follow-up. Arthroscopy 29: 2020-2028, 2013.

2. Gopal K, Amirhamed HA and Kamarul T: Advances of human bone marrow-derived mesenchymal stem cells in the treatment of cartilage defects: a systematic review. Exp Biol Med (Maywood) 239: 663-669, 2014.

3. Granado-Serrano AB, Martín MA, Izquierdo-Pulido M, et al: Molecular mechanisms of (-)-epicatechin and chlorogenic acid on the regulation of the apoptotic and survival/proliferation pathways in a human hepatoma cell line. J Agric Food Chem 55: 2020-2027, 2007.

4. Wan CW, Wong CN, Pin WK, et al: Chlorogenic acid exhibits cholesterol lowering and fatty liver attenuating properties by up-regulating the gene expression of PPAR- $\alpha$ in hypercholesterolemic rats induced with a high-cholesterol diet. Phytother Res 27: 545-551, 2013.

5. Li S, Bian H, Liu Z, et al: Chlorogenic acid protects MSCs against oxidative stress by altering FOXO family genes and activating intrinsic pathway. Eur J Pharmacol 674: 65-72, 2012.

6. Feng GS: Shp2-mediated molecular signaling in control of embryonic stem cell self-renewal and differentiation. Cell Res 17: 37-41, 2007.

7. Nishida $\mathrm{K}$ and Hirano $\mathrm{T}$ : The role of Gab family scaffolding adapter proteins in the signal transduction of cytokine and growth factor receptors. Cancer Sci 94: 1029-1033, 2003.

8. Lee TI, Jenner RG, Boyer LA, et al: Control of developmental regulators by Polycomb in human embryonic stem cells. Cell 125: 301-313, 2006.

9. Craddock BL, Hobbs J, Edmead CE and Welham MJ: Phosphoinositide 3-kinase-dependent regulation of interleukin-3-induced proliferation: involvement of mitogen-activated protein kinases, SHP2 and Gab2. J Biol Chem 276: 24274-24283, 2001.

10. Gharibi B, Abraham AA, Ham J and Evans BA: Contrasting effects of $\mathrm{A} 1$ and $\mathrm{A} 2 \mathrm{~b}$ adenosine receptors on adipogenesis. Int JObes (Lond) 36: 397-406, 2012.

11. Wu L, Cai X, Dong H, et al: Serum regulates adipogenesis of mesenchymal stem cells via MEK/ERK-dependent PPARgamma expression and phosphorylation. J Cell Mol Med 14: 922-932, 2010.

12. Bai X, Ma D, Liu A, et al: Rheb activates mTOR by antagonizing its endogenous inhibitor, FKBP38. Science 318: 977-980, 2007.

13. Han Y, Caday CG, Nanda A, et al: Tyrphostin AG 1478 preferentially inhibits human glioma cells expressing truncated rather than wild-type epidermal growth factor receptors. Cancer Res 56: 3859-3861, 1996. 
14. Zhan Y, Counelis GJ and O'Rourke DM: The protein tyrosine phosphatase SHP-2 is required for EGFRvIII oncogenic transformation in human glioblastoma cells. Exp Cell Res 315: 2343-2357, 2009.

15. Livak KJ and Schmittgen TD: Analysis of relative gene expression data using real-time quantitative PCR and the 2(-Delta Delta C(T)) method. Methods 25: 402-408, 2001.

16. Wu CJ, O'Rourke DM, Feng GS, Johnson GR, Wang Q and Greene MI: The tyrosine phosphatase SHP-2 is required for mediating phosphatidylinositol 3-kinase/Akt activation by growth factors. Oncogene 20: 6018-6025, 2001.

17. Zhang HH, Huang J, Düvel K, et al: Insulin stimulates adipogenesis through the Akt-TSC2-mTORC1 pathway. PLoS One 4: e6189, 2009.

18. Wang T, Wang Y and Yamashita H: Evodiamine inhibits adipogenesis via the EGFR-PKCalpha-ERK signaling pathway. FEBS Lett 583: 3655-3659, 2009.

19. Roobrouck VD, Ulloa-Montoya F and Verfaillie CM: Self-renewal and differentiation capacity of young and aged stem cells. Exp Cell Res 314: 1937-1944, 2008.

20. Zheng H, Ying H, Wiedemeyer R, et al: PLAGL2 regulates Wnt signaling to impede differentiation in neural stem cells and gliomas. Cancer Cell 17: 497-509, 2010.

21. Liu HK, Wang Y, Belz T, et al: The nuclear receptor tailless induces long-term neural stem cell expansion and brain tumor initiation. Genes Dev 24: 683-695, 2010.

22. Liu L and Rando TA: Manifestations and mechanisms of stem cell aging. J Cell Biol 193: 257-266, 2011.

23. Zhu X, Zhang H and Lo R: Phenolic compounds from the leaf extract of artichoke (Cynara scolymus L.) and their antimicrobial activities. J Agric Food Chem 52: 7272-7278, 2004.

24. Jiang F and Dusting GJ: Natural phenolic compounds as cardiovascular therapeutics: potential role of their antiinflammatory effects. Curr Vasc Pharmacol 1: 135-156, 2003.

25. Johnston KL, Clifford MN and Morgan LM: Coffee acutely modifies gastrointestinal hormone secretion and glucose tolerance in humans: glycemic effects of chlorogenic acid and caffeine. Am J Clin Nutr 78: 728-733, 2003.

26. Karthikesan K, Pari L and Menon VP: Antihyperlipidemic effect of chlorogenic acid and tetrahydrocurcumin in rats subjected to diabetogenic agents. Chem Biol Interact 188: 643-650, 2010.
27. Zheng Q, Hirose Y, Yoshimi N, et al: Further investigation of the modifying effect of various chemopreventive agents on apoptosis and cell proliferation in human colon cancer cells. J Cancer Res Clin Oncol 128: 539-546, 2002.

28. Jiang Y, Kusama K, Satoh K, et al: Induction of cytoxicity by chlorogenic acid in human oral tumor cell lines. Phytomedicine 7: 483-491, 2000

29. Hsu CL, Huang SL and Yen GC: Inhibitory effect of phenolic acids on the proliferation of 3T3-L1 preadipocytes in relation to their antioxidant activity. J Agric Food Chem 54: 4191-4197, 2006.

30. Zaragosi LE, Ailhaud G and Dani C: Autocrine fibroblast growth factor 2 signaling is critical for self-renewal of human multipotent adipose-derived stem cells. Stem Cells 24: 2412-2419, 2006.

31. Makino T, Jinnin M, Muchemwa FC, et al: Basic fibroblast growth factor stimulates the proliferation of human dermal fibroblasts via the ERK1/2 and JNK pathways. Br J Dermatol 162: 717-723, 2010.

32. Choi SC, Kim SJ, Choi JH, et al: Fibroblast growth factor-2 and -4 promote the proliferation of bone marrow mesenchymal stem cells by the activation of the PI3K-Akt and ERK1/2 signaling pathways. Stem Cells Dev 17: 725-736, 2008.

33. GharibiB1, Ghuman MS and Hughes FJ: Akt-andErk-mediated regulation of proliferation and differentiation during PDGFR $\beta$-induced MSC self-renewal. J Cell Mol Med 16: 2789-2801, 2012.

34. Zhang W, Shen X, Wan C, Zhao Q, Zhang L, Zhou Q and Deng L: Effects of insulin and insulin-like growth factor 1 on osteoblast proliferation and differentiation: differential signalling via Akt and ERK. Cell Biochem Funct 30: 297-302, 2012.

35. Wu C, Luan $\mathrm{H}$, Zhang X, et al: Chlorogenic acid protects against atherosclerosis in ApoE-/- mice and promotes cholesterol efflux from RAW264.7 macrophages. PLoS One 9: e95452, 2014.

36. Fritzius $\mathrm{T}$ and Moelling K: Akt- and Foxol-interacting WD-repeat-FYVE protein promotes adipogenesis. EMBO J 27: 1399-1410, 2008.

37. Hinoi E, Iezaki T, Fujita H, Watanabe T, Odaka Y, Ozaki K and Yoneda Y: PI3K/Akt is involved in brown adipogenesis mediated by growth differentiation factor-5 in association with activation of the Smad pathway. Biochem Biophys Res Commun 450: 255-260, 2014.

38. Wang M, Wang JJ, Li J, et al: Pigment epithelium-derived factor suppresses adipogenesis via inhibition of the MAPK/ERK pathway in 3T3-L1 preadipocytes. Am J Physiol Endocrinol Metab 297: E1378-E1387, 2009. 